\title{
Cost-effectiveness analysis of the introduction of S-1 therapy for first-line metastatic breast cancer treatment in Japan: results from the randomized phase III SELECT BC trial
}

Takeru Shiroiwa ${ }^{*}$, Takashi Fukuda', Kojiro Shimozuma², Mitsuko Mouri ${ }^{3}$, Yasuhiro Hagiwara ${ }^{4}$, Takuya Kawahara ${ }^{4,5}$, Shozo Ohsumi ${ }^{6}$, Yasuo Hozumi ${ }^{7,8}$, Yoshiaki Sagara ${ }^{9}$, Yasuo Ohashi ${ }^{10}$ and Hirofumi Mukai ${ }^{11}$

\begin{abstract}
Background: This study evaluated the cost-effectiveness of replacing standard intravenous therapy (taxane) with oral S-1 therapy for first-line metastatic breast cancer treatment.

Methods: This cost-effectiveness analysis was based on data from a randomized phase III trial (SELECT BC). As cost-effectiveness was a secondary endpoint of the SELECT BC trial, some of the randomized patients participated in an EQ-5D survey $(N=391)$ and health economic survey $(N=146)$. The EQ-5D responses, claims, and prescription data were collected for as long as possible until death. The expected quality-adjusted life years (QALY) obtained from each treatment were calculated using patient-level EQ-5D data, and the expected cost was calculated using patient-level claim data. The analysis was performed from the perspective of public healthcare payers.
\end{abstract}

Results: The estimated EQ-5D least-square means and $95 \% \mathrm{Cl}$ up to 48 months were $0.764(95 \% \mathrm{Cl}, 0.741-0.782)$ and 0. $742(95 \% \mathrm{Cl}, 0.720-0.764)$ in the S-1 and taxane arms, respectively. The expected QALY was 2.11 for the S-1 arm and 2. 04 for the taxane arm, with expected costs of JPY 5.13 million (USD 46,600) and JPY 5.56 million (USD 50,500), respectively. These results show that S-1 is cost-saving. According to probabilistic sensitivity analysis, S-1 was dominant with a probability of 63\%. When the willingness to pay (WTP) value was JPY 5 million (USD 45,500) per QALY, the probability of being cost-effective was $92 \%$.

Conclusions: Our results show that the introduction of oral S-1 therapy for metastatic breast cancer is highly likely to be cost-effective.

Trial registration: UMIN CTR C000000416. Registered on May 10, 2006.

Keywords: Cost-effectiveness analysis, Quality-adjusted life years, Breast neoplasms, Randomized controlled trial, S-1, Taxoids

\footnotetext{
*Correspondence: t.shiroiwa@gmail.com; shiroiwa@niph.go.jp

${ }^{1}$ Department of Health and Welfare Services, National Institute of Public

Health, 2-3-6 Minami, Wako, Saitama 351-0197, Japan

Full list of author information is available at the end of the article
} 


\section{Background}

A number of novel anticancer drugs have been developed this decade, leading to a gradual improvement in the outcomes of cancer patients. However, the economic influence of these drugs on current public medical expenditures has become substantial due to their relatively high prices. Under these circumstances, and with the present budget constraints in healthcare, it is important to consider not only the safety and efficacy, but also the cost-effectiveness of anticancer drugs. In fact, many health technology assessment (HTA) organizations focus on new innovative anticancer drugs. For example, the National Institute for Health and Care Excellence (NICE) in the UK began evaluating all anticancer drugs in 2016 [1, 2] with the reform of cancer drugs fund. Some HTA agencies (e.g., NICE, the pan-Canadian Oncology Drug Review (pCODR) in Canada, and the Pharmaceutical Benefits Advisory Committee (PBAC) in Australia) concluded that certain chemotherapy regimens are not cost-effective and should not be recommended for routine use under the public healthcare system [3-5].

S-1 [6] (tegafur with gimeracil and oteracil, Teysuno $\%$ TS $\left.-1^{\circ}\right)$ is an oral fluoropyrimidine anticancer drug that does not require intravenous administration, unlike many other chemotherapy agents. Thus, patients receiving oral S-1 therapy do not need to bear long hours of intravenous administration and adverse events (e.g. phlebitis) associated with intravenous administration. In addition, a hospital visit is required to receive chemotherapy whenever intravenous anticancer drugs are administered. Therefore, S-1 may not only provide a convenient option for metastatic breast cancer (MBC) therapy, but may also improve the efficiency of treatment. S-1 has been approved in some Asian countries (Japan, Korea, Mainland China, Singapore, Taiwan, etc.) and European countries (UK, Germany, Sweden, etc.) for gastric cancer. However, Japan is the first country to avail S-1 to $\mathrm{MBC}$ patients.

SELECT BC [7] is a phase III, open-label, randomized controlled trial (RCT) that compared S-1 with taxanes (paclitaxel or docetaxel) for first-line MBC therapy. According to treatment algorithms (Hortobagyi [8] and NCCN guidelines [9]), patients irresponsive to endocrine therapy receive cytotoxic chemotherapy in standard cases. Taxanes are among the first-choice chemotherapy agents for $\mathrm{MBC}$ patients. However, the trial demonstrated non-inferiority of $\mathrm{S}-1$ to taxane in overall survival (OS), with a median OS of 37.2 months in the taxane arm vs. 35.0 months in the S-1 arm (hazard ratio (HR) 1.05, 95\% CI 0.86-1.27, $p=0.015$ ), at a median follow-up of 34.6 months. The SELECT BC trial also evaluated cost-effectiveness as a secondary endpoint in addition to some clinical endpoints, including health-related quality of life (HRQOL).
In the SELECT BC trial, EuroQol 5-dimension (EQ-5D) measurements $[10,11]$ and claims (receipt) data collection for economic evaluation were also performed. These longitudinal patient-level EQ-5D and claims data can be used to calculate quality-adjusted life years (QALYs) and medical costs for evaluation of long-term cost-effectiveness. Such a trial-based $[12,13]$ cost-effectiveness analysis could improve the robustness of analysis and validity of internal comparison compared to a model-based approach [14] (e.g., using Markov model [15]). In this paper, we report on a cost-effectiveness analysis of the introduction of S-1 therapy to first-line $\mathrm{MBC}$ treatment using data from the SELECT BC trial.

\section{Methods}

\section{Study design}

In the SELECT BC trial, patients with HER2-negative, hormone-resistant $\mathrm{MBC}$ who were not previously treated with chemotherapy after diagnosis were randomized at a 1:1 ratio and allocated to the taxane arm (docetaxel 60$75 \mathrm{mg} / \mathrm{m}^{2} \mathrm{q} 3 \mathrm{w}$, paclitaxel $80-100 \mathrm{mg} / \mathrm{m}^{2} \mathrm{q} 1 \mathrm{w}$, or paclitaxel $175 \mathrm{mg} / \mathrm{m}^{2} \mathrm{q} 3 \mathrm{w}$ at the discretion of the treating physician) or S-1 arm (40-60 mg twice daily based on the patient's body surface area, for 28 days on and 14 days off). Treatment continued until the disease progressed or more than four cycles of S-1 or six cycles of taxane were administered.

The enrollment period of the SELECT BC trial was from October 2006 to July 2010, and the trial involved 154 institutions in Japan. HRQOL was assessed using two instruments: the European Organization for Research and Treatment of Cancer Core Quality of Life Questionnaire C30 (EORTC QLQ-C30) [16] and EQ-5D. Not all 618 randomized patients responded to the HRQOL instruments; selection of HRQOL respondents was based on each institution. Some institutions were excluded in advance due to feasibility issues. Claims data were collected from a portion of the HRQOL population for the same reason. As institution was a prognostic factor for dynamic allocation, patient background factors were expected to be balanced in both arms.

The study was conducted in accordance with the Ethical Guidelines for Clinical Research of the Japanese Ministry of Health, Labour and Welfare and the Declaration of Helsinki. Written informed consent was obtained from each participant. Approval for the protocol and any modifications was obtained from an independent ethics committee of each participating institution. The SELECT BC trial was prospectively registered with the University Hospital Medical Information Network (UMIN) in Japan (protocol ID C000000416).

\section{EQ-5D assessment and claims data collection}

EQ-5D is the most commonly used preference-based measure for assessing HRQOL $[17,18]$. It can be used 
to calculate QALYs for the economic evaluation of healthcare technologies. We used the EQ-5D 3-level version, which comprises five items: "mobility," "self-care," "usual activities," "pain/discomfort," and "anxiety/depression," at three levels of description. Responses can be converted to an EQ-5D score using a predetermined algorithm based on societal preferences of the general population [11].

In the SELECT BC trial, EQ-5D measurements were continued over a long period because measurements could be continued even when the disease progressed. Collection of monthly claims data was also continued to estimate treatment costs in the same manner. Patients responded to the Japanese version of the EQ-5D [11] at baseline and at 3, 6, and 12 months, and every 6 months thereafter until death or to the extent possible. In general, patients responded to the EQ-5D before the next cycle of chemotherapy was administered.

Claims data were created monthly by each institution for reimbursement of medical costs through public medical insurance in Japan. Claims data included all items of medical resources and drugs consumed in an institution, including those for adverse events. In addition, information on amounts and costs of each consumed item were included. We directly collected them from each institution, deleting patients' personal information. However, claims data contained no information regarding pharmacy prescriptions. Accordingly, we also collected prescriptions from each institution. As claims data are not created by institutions when the patient's monthly medical expenses were 0 , we cannot distinguish whether the absence of claims data means no costs or missing data. Our data center contacted institutions to confirm whether no submission of claims data indicated no costs or missing data.

\section{Frameworks of cost-effectiveness analysis}

We performed a cost-effectiveness analysis from the perspective of public healthcare payers. The time horizon was limited to 4 years, which is considered long enough to evaluate the values of healthcare technologies, given the quantity of collected claims data. The Japanese Breast Cancer Society clinical practice guidelines in 2013 recommended the use of anthracycline- or taxane-based regimens as first-line therapy for HER2 negative MBC patients. In 2015, the guidelines were revised to include S-1 in the recommended first-line therapies for HER2 negative patients [19] based on the results of the SELECT BC trial. Therefore, we selected taxanes as a comparator because taxanes are one of the standard therapies for first-line HER2 negative patients. The Japanese methodological guidelines for economic evaluation [20] recommend a $2 \%$ discount rate; therefore, cost and effectiveness was discounted by $2 \%$ per year, and the rate was changed from $0 \%$ to $4 \%$ as a sensitivity analysis in accordance with the guidelines. Unit costs were based on the Japanese fee schedule and drug tariff as of 2016, both of which are defined by the Ministry of Health, Labour and Welfare at an exchange rate of USD $1=$ JPY 110 as of May 2016, as reported by the Bank of Japan.

The planned sample populations for the HRQOL analysis and cost analysis was approximately 300 and 150, respectively; these numbers were not based on a statistical calculation because HRQOL and cost-effectiveness in the SELECT BC trial were not the confirmatory endpoints. Collected responses were converted to EQ-5D index values [11].

Health outcomes of each intervention are evaluated in QALY. The expected QALY obtained from each treatment was calculated using patient-level data on survival and EQ-5D. Linear mixed models for repeated measures (MMRM) were applied to estimate EQ-5D scores. EQ5D scores were adjusted by baseline score, treatment, time, and treatment-by-time interaction. Patient individual effect was also added to the model as a random effect. Responses with more than one missing items were treated as missing values, and they were analyzed based on "missing at random" assumption without any implementation. Estimates of the least-square means for EQ-5D score and 95\% confidence intervals (CIs) were calculated by each visit and group. QALY between visits at $t_{i}$ month and $t_{i+1}$ month was calculated by $\operatorname{OS}\left(t_{i}\right) * 1 /$ $2\left(\operatorname{EQ} 5 D\left(t_{i}\right)+\operatorname{EQ5D}\left(t_{i+1}\right)\right) *\left(t_{i+1}-t_{i}\right)$, using the estimated EQ-5D values. The expected cost (i.e., sum of costs from claims and prescription data) was calculated using patient-level survival and claims/prescription data by Lin's method [21]; mean costs between visit (= total cost / number of observed patients) were multiplied by Kaplan-Meier estimator. If no claims data were collected, treatment costs for the corresponding month were treated as 0 , unless claims data were no longer collected in future months. After the final claims data were received, subsequent data (until death) were censored.

Using estimates for expected costs and outcomes (QALY), incremental cost-effectiveness ratio (ICER) was calculated if superiority of EQ-5D values or OS (i.e., positive incremental effective value) was shown. However, it was clearly revealed that we could not expect superiority in HRQOL and OS. In such cases, if additional benefit could not be demonstrated, only the costs of both groups were compared based on the so-called "cost-minimization" approach in base-case analysis. The Bootstrap method (10,000 times resampling) was used for probabilistic sensitivity analysis, and a costeffectiveness acceptability curve was created [22]. Unlike base-case analysis, the ICER may be calculated in each simulation [23]. 
As a scenario analysis, we adjusted drug costs by current drug prices as of May 2016. In Japan, drug prices generally decrease every 2 years based on the actual market price, with some exceptions. In addition, generics of taxane and S-1 are already in the market (breast cancer is not an indication for generics of S-1 yet, but S-1 for breast cancer will be off-patent in a few years). We also performed an analysis on generics by replacing taxane and S-1 with their average generic prices as of 2017, e.g., JPY 372.5 (USD 3.4) [S-1 $25 \mathrm{mg}$ capsule], JPY 14,798 (USD 134.5) [paclitaxel $100 \mathrm{mg}$ vial], and JPY 29,802 (USD 270.9) [docetaxel $80 \mathrm{mg}$ vial]. All analyses were performed with $\mathrm{SAS}^{\circ}$ 9.4 and R 3.3.1.

\section{Results}

\section{Patient population}

Participants were 618 Japanese $\mathrm{MBC}$ patients randomly assigned to either the taxane $(N=309)$ or S-1 $(N=309)$ arm. A total of 175 and 208 patients in the taxane and S-1 arms, respectively, were included in the sample population for the HRQOL analysis. In the taxane arm, 96 patients received docetaxel and 79 received paclitaxel. Among patients subject to the cost analysis, 70 were allocated to the taxane (41 docetaxel and 29 paclitaxel) arm and 76 to the S-1 arm. Baseline characteristics of the patients were balanced between the two arms (Table 1) with similar distributions evident across the whole full analysis set (FAS) population.

\section{Completion rates of EQ-5D and the quantity of collected claims data}

Longitudinal EQ-5D completion rates and the number of patients with collected claims data are shown in Table 2. The mean duration of EQ-5D measurements was 21 months for both groups. Completion rates at 3 months were $88.3 \%$ and $83.6 \%$ in the taxane and S-1 arms, respectively, and $71.8 \%$ and $77.6 \%$, respectively, at 12 months. Although the percentage gradually declined with time, more than half of the patients completed the instrument up to 48 months. On the other hand, according to the record of the data center, the collection rate of claims data was roughly $100 \%$. Thus, indications of no collected claims data should be interpreted as zero medical costs instead of missing data.

\section{Cost-effectiveness of S-1 therapy}

The longitudinal scores of the EQ-5D are shown in Fig. 1. The estimated EQ-5D least-square means and

Table 1 Patient demographics

\begin{tabular}{|c|c|c|c|c|c|c|}
\hline \multirow[b]{4}{*}{ Median age } & \multicolumn{2}{|c|}{ QOL population } & \multicolumn{2}{|c|}{ Cost population } & \multicolumn{2}{|c|}{ FAS population } \\
\hline & Taxane & S-1 & Taxane & S-1 & Taxane & $\mathrm{S}-1$ \\
\hline & $N=175$ & $N=208$ & $N=70$ & $N=76$ & $N=286$ & $N=306$ \\
\hline & 57.0 & 59.0 & 58.0 & 59.0 & 58.5 & 59.0 \\
\hline \multicolumn{7}{|l|}{ Hormone receptor status } \\
\hline ER-positive, PgR-positive, or both & $127(72.6)$ & $149(71.6)$ & $50(71.4)$ & $54(71.1)$ & $212(74.1)$ & $223(72.9)$ \\
\hline ER-negative and PgR-negative & $45(25.7)$ & $53(25.5)$ & $18(25.7)$ & $20(26.3)$ & $71(24.8)$ & $76(24.8)$ \\
\hline Unknown & $3(1.7)$ & $6(2.9)$ & $2(2.9)$ & $2(2.6)$ & $3(1.0)$ & $7(2.3)$ \\
\hline \multicolumn{7}{|l|}{ HER2 status } \\
\hline Negative & $162(92.6)$ & $192(92.3)$ & $64(91.4)$ & $71(93.4)$ & $264(92.3)$ & $282(92.2)$ \\
\hline Unknown & $13(7.4)$ & $16(7.7)$ & $6(8.6)$ & $5(6.6)$ & $22(7.7)$ & $24(7.8)$ \\
\hline \multicolumn{7}{|c|}{ Components of (neo)adjuvant treatment } \\
\hline Oral fluoropyrimidine & $26(14.9)$ & $22(10.6)$ & $9(12.9)$ & $10(13.2)$ & 39 (13.6) & $35(11.4)$ \\
\hline Taxane & $49(28.0)$ & $61(29.3)$ & $19(27.1)$ & $24(31.6)$ & $80(28.0)$ & $80(26.1)$ \\
\hline Endocrine therapy & $100(57.1)$ & $111(53.4)$ & $44(62.9)$ & $45(59.2)$ & $170(59.4)$ & $169(55.2)$ \\
\hline \multicolumn{7}{|l|}{ Disease-free interval } \\
\hline$\leq 2$ years & $34(19.4)$ & $41(19.7)$ & $14(20.0)$ & $19(25.0)$ & $57(19.9)$ & $60(19.6)$ \\
\hline $2-5$ years & $52(29.7)$ & $66(31.7)$ & $23(32.9)$ & $22(28.9)$ & $98(34.3)$ & $103(33.6)$ \\
\hline$\geq 5$ years & $58(33.1)$ & $67(32.2)$ & $24(34.3)$ & $25(32.9)$ & $86(30.0)$ & $94(30.7)$ \\
\hline Unknown & $0(0.0)$ & $0(0.0)$ & $0(0.0)$ & $0(0.0)$ & $0(0.0)$ & $2(0.7)$ \\
\hline No surgery & $31(17.7)$ & $34(16.3)$ & $9(12.9)$ & $10(13.2)$ & $45(15.7)$ & $47(15.4)$ \\
\hline \multicolumn{7}{|l|}{ Liver metastasis } \\
\hline Yes & $61(34.9)$ & $78(37.5)$ & $20(28.6)$ & 27 (35.5) & 96 (33.6) & $103(33.7)$ \\
\hline No & $114(65.1)$ & $130(62.5)$ & $50(71.4)$ & 49 (64.5) & $190(66.4)$ & $203(66.3)$ \\
\hline
\end{tabular}


Table 2 Collection rate of EQ-5D and claims data

\begin{tabular}{|c|c|c|c|c|}
\hline & \multicolumn{2}{|l|}{ QOL population } & \multicolumn{2}{|c|}{ Cost population } \\
\hline & Taxane & $S-1$ & Taxane & $\mathrm{S}-1$ \\
\hline & $N=175$ & $N=208$ & $N=70$ & $N=76$ \\
\hline Baseline/Month 1 & 175/175 (100) & 208/208 (100) & 54 & 66 \\
\hline Month 3 & 151/171 (88.3) & 168/201 (83.6) & 70 & 70 \\
\hline Month 6 & $138 / 168(82.1)$ & 146/190 (76.8) & 66 & 66 \\
\hline Month 12 & 107/149 (71.8) & 132/170 (77.6) & 49 & 56 \\
\hline Month 18 & 75/126 (59.5) & 107/158 (67.7) & 41 & 45 \\
\hline Month 24 & $68 / 117(58.1)$ & 93/137 (67.9) & 38 & 45 \\
\hline Month 30 & $51 / 101(50.5)$ & $68 / 110(61.8)$ & 33 & 35 \\
\hline Month 36 & 45/90 (50.0) & 47/84 (56.0) & 32 & 27 \\
\hline Month 42 & 27/61 (44.3) & $31 / 61(50.8)$ & 29 & 14 \\
\hline Month 48 & 18/39 (46.2) & 21/37 (56.8) & 18 & 15 \\
\hline
\end{tabular}

95\% CI up to 48 months were 0.764 (95\% CI, $0.741-$ 0.782 ) and 0.742 (95\% CI, $0.720-0.764)$ in the S-1 and taxane arms, respectively (Appendix). EQ-5D values in the S-1 arm were not significantly larger than those in the taxane arm. The expected QALY was 2.11 for the S1 arm and 2.04 for the taxane arm, while the expected costs were JPY 5.13 million (USD 46,600) and JPY 5.56 million (USD 50,500), respectively (Table 3). S-1 therapy was cost-saving by JPY 0.43 million (USD 3900) [SE: JPY 0.4 million], with increased QALY by 0.07 [SE: 0.09]. When OS data were extrapolated using Weibull regression analysis, the expected QALYs were approximately the same for S-1 (2.48) and taxane (2.50) arms.

According to the sensitivity analysis of the discount rate from $0 \%$ to $4 \%$, incremental costs were not changed from PY 0.43 million (USD 3900). In the S-1 arm, outpatient cost was JPY 3.52 million (USD 32000), and inpatient cost was JPY 1.61 million (USD 14,600). In the taxane arm, outpatient cost was JPY 4.07 (USD 37,000), and inpatient cost was JPY 1.49 million (USD 13,500).

These results suggest that the S-1 arm obtained more QALYs at lower costs; i.e., that this option was dominant. According to probabilistic sensitivity analysis, the cost-effectiveness acceptability curve and scatter plot are presented in Fig. 2. The figure shows S-1 was dominant with a probability of $63 \%$ if the time horizon was limited to 4 years. When the willingness to pay (WTP) value was JPY 5 million (USD 45,500) per QALY [24], the probability of being cost-effective was $92 \%$.

If drug prices were adjusted to current rates, the costs for both groups decreased to JPY 4.50 million (USD 40,900 ) in the S-1 arm and JPY 4.78 million (USD $43,400)$ in the taxane arm. In the S-1 and taxane arms, drug costs were JPY 1.14 million (USD 10,300) and JPY 1.48 million (USD 13,400), respectively.

The percentage of drug costs calculated by each of the four digits of the WHO-ATC code [25] was obtained (Table 4). The costs of L01 (antineoplastic agents) accounted roughly for more than $50 \%$ of drug costs.

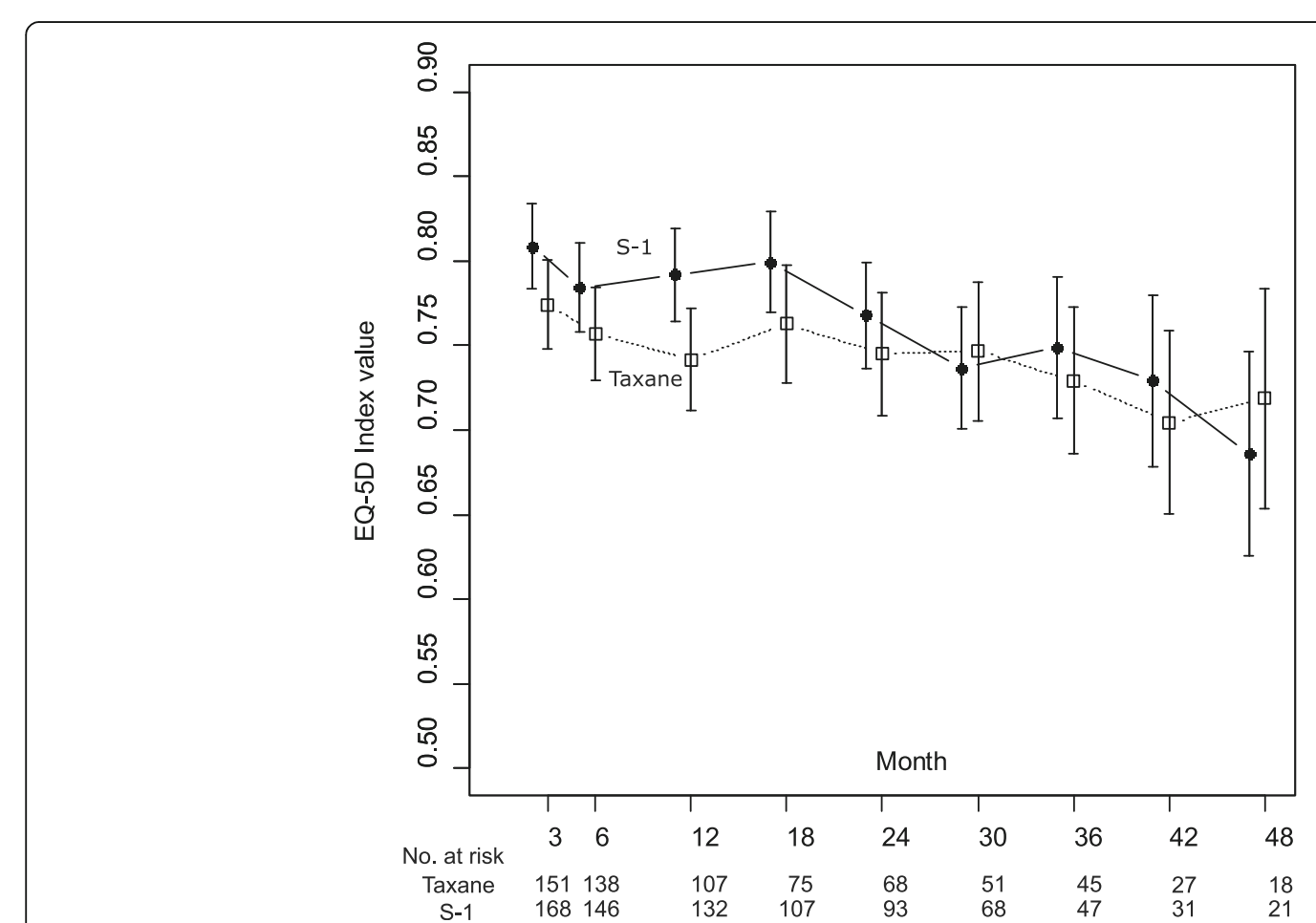

Fig. 1 Longitudinal EQ-5D index. *Footnote of Fig. 1: This figure shows estimates of the least-square means for EQ-5D value and 95\% confidence intervals. Black circle indicates values of S-1 group, and white square does those of taxane group 
Table 3 Results of cost-effectiveness analysis

\begin{tabular}{llclc}
\hline Group & E (QALY) & IE (QALY) & C (JPY 1000) & IC (JPY 1000) \\
\hline S-1 & 2.11 & 0.070 & 5307 & -424 \\
& & & [USD 47,000] & [USD 3750] \\
Taxane & 2.04 & & 5731 & \\
& & & [USD 50,700] & \\
\hline
\end{tabular}

$E$ Effectiveness, IE Incremental effectiveness, C Cost, IC Incremental cost

About $10-15 \%$ of drug costs were for M05 (drugs for treatment of bone diseases), into which mainly bisphosphonates for bone metastasis were classified. Analgesics (N02), endocrine therapy (L02), and antiemetics and antinauseants (A04) accounted for less than 10\% of total drug costs.

Furthermore, when taxane and S-1 were replaced by generics, the cost of S-1 was JPY 4.16 million (USD 37,900), and taxane was JPY 4.39 million (USD 39,900).
The cost difference between S-1 and taxane diminished if both taxane and S-1 were completely replaced by generics. When the price of generic S-1 was increased by more than 2.3 times, the cost of taxane was smaller than that of S-1.

\section{Discussion}

We performed a cost-effectiveness analysis of oral S-1 therapy for $\mathrm{MBC}$ patients. The analysis was mainly based on information (survival, QOL, and treatment costs) collected from the Phase III randomized SELECT $\mathrm{BC}$ trial. Our results suggest that $\mathrm{S}-1$ is cost-saving and the probability of being dominant (i.e., superior in both effectiveness and costs) is high compared with standard taxane therapy. A number of economic evaluations concluded that some anticancer drugs are either not costeffective or have increased treatment costs even if they

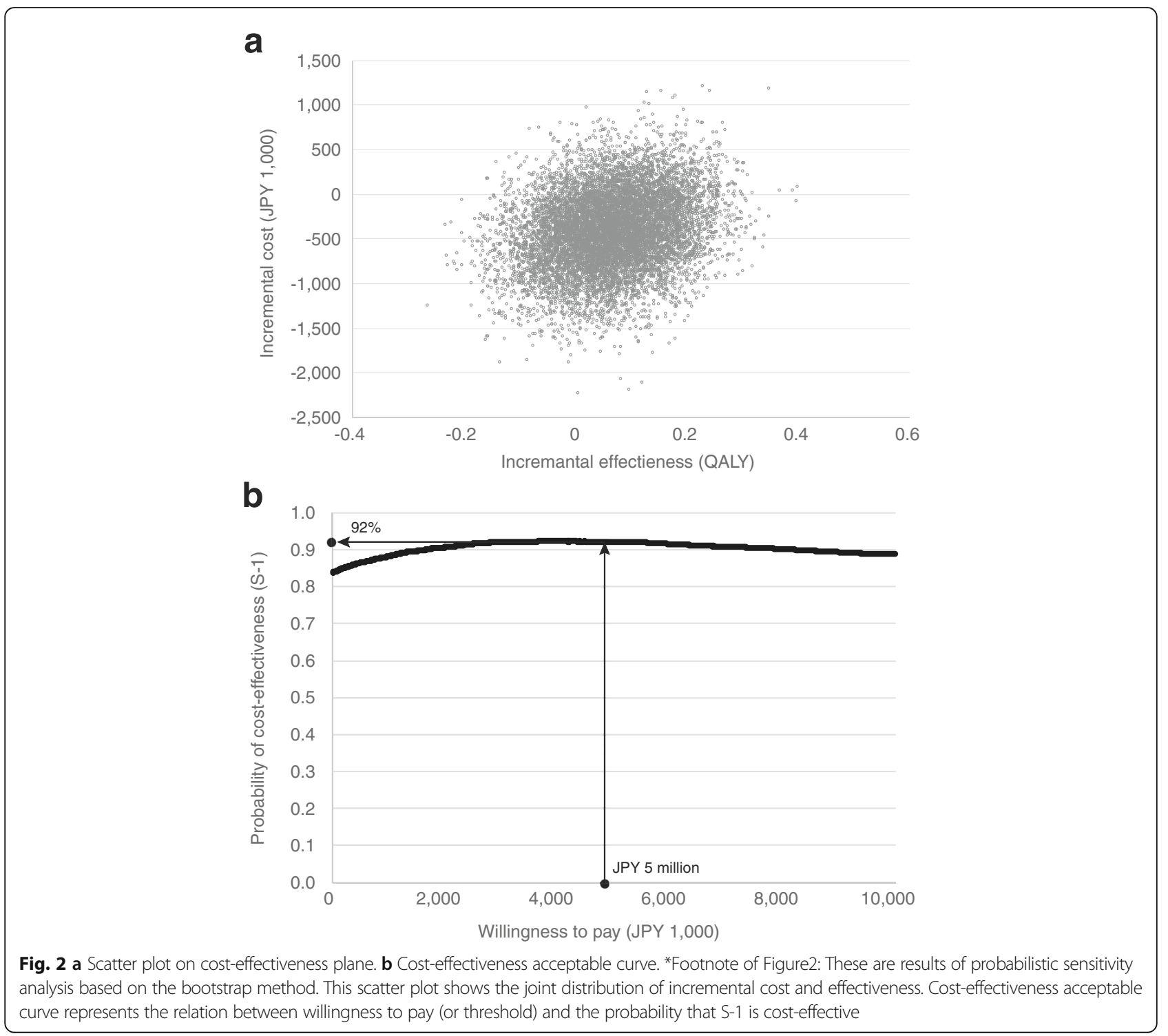


Table 4 Percentage of drug costs classified by ATC

\begin{tabular}{llll}
\hline ATC code & ATC name & Taxane & S-1 \\
\hline L01 & Antineoplastic agents & $66 \%$ & $59 \%$ \\
M05 & Drugs for treatment of bone diseases & $10 \%$ & $16 \%$ \\
N02 & Analgesics & $9 \%$ & $3 \%$ \\
L02 & Endocrine therapy & $3 \%$ & $9 \%$ \\
A04 & Antiemetics and antinauseants & $3 \%$ & $2 \%$ \\
V08 & Contrast media & $2 \%$ & $2 \%$ \\
V09 & Diagnostic raidopharmaceutical & $1 \%$ & $1 \%$ \\
A02 & Drugs for acid and related disorders & $1 \%$ & $1 \%$ \\
& Others & $5 \%$ & $7 \%$ \\
\hline
\end{tabular}

are cost-effective. However, our results revealed a high probability that $\mathrm{S}-1$ therapy is cost-saving or dominant with high probability. Considering these results, S-1 may become one of the standard therapies used to treat MBC patients.

This study used Japanese unit costs (e.g., acquisition costs and drug prices) to estimate expected costs of chemotherapy, and our results cannot be simply extrapolated to other countries. However, in Europe, although S-1 has not yet been approved for $\mathrm{MBC}$, the introduction of S-1 therapy for MBC patients may have larger economic effects because the difference in drug costs between the $\mathrm{S}-1$ and taxane group was larger in both the UK and Germany than Japan. According to the British National Formulary (BNF) and Rote Liste, a $20 \mathrm{mg}$ capsule of S-1 is JPY 564.7 (USD 5.1) in Japan, GBP 2.96 (USD 3.7, GBP $1=$ USD 1.26) in UK and EUR 6.01 (USD 6.5, 1 EUR $=$ USD 1.07) in Germany. For Docetaxel $\left(\right.$ Taxotel $^{\circ}$ ), an $80 \mathrm{mg}$ vial is JPY 52,835 (USD 480) in Japan, GBP 504.27 (USD 635) and EUR 783.17 (USD 838) in Germany. A $100 \mathrm{mg}$ vial of Paclitaxel is JPY 22,071 (USD 201) in Japan, GBP 200.35 (USD 252) in UK and EUR 400.57 (USD 428.6) [the lowest price] in Germany. If the drug prices of $S-1$ and taxane in the UK were applied, the cost would be GBP 6200 (USD 7810) for S-1 and GBP 9310 (USD $11,700)$ for taxane. Similarly, drug cost as calculated by German pricing was EUR 11,900 (USD 12,700) for S-1 and EUR 16200 (USD 17,300) for taxane. Differences in drug costs between groups in the UK and Germany were larger than those in Japan, because the list price of taxane in the UK and Germany is higher than Japan; conversely, the cost of $\mathrm{S}-1$ is similar or lower.

Our previous study examined longer-term (60 months) EQ-5D index values [26] and reported that the values were higher in the S-1 arm than the taxane arm when the analysis was limited to the first 12 months during progression-free survival (PFS).
However, the values did not differ between arms when observations were continued up to 60 months. In the present evaluation, a 48-month analysis was performed to conform to the time horizon of costeffectiveness analysis, although the above descriptions are also applied to the results of the EQ-5D in this analysis. This suggests that EQ-5D values of S-1 might be higher than those of taxane when patients receive chemotherapy. However, the difference was not statistically significant due to variation in EQ-5D values after chemotherapy, which are longer and more influential toward the results. In fact, the scores of EORTC QLQ-C30 were higher in the S-1 arm than in the taxane arm during 12 months from randomization for global health state (by $4.5 ; p=$ 0.039), as well as for all five functional domains including physical functioning, role functioning, emotional functioning, cognitive functioning, and social functioning [7].

In this study, the HRQOL and costs population comprised only a portion of the whole population. Only patients from contacted institutions completed the survey on HRQOL and costs. This design came about after considering the feasibility that some organization could not collect these data because of human resource restraints (e.g. lack of a clinical research coordinator at small institutions). Of course, while this design may have also caused potential selection bias of patients, institution was one of the adjusted factors for allocation in the SELECT BC trial. As shown in Table 1, the background factors of QOL and cost population were comparable to those of the whole FAS population. While the study design may be one limitation of the present investigation, we also feel that our results maintained high internal validity.

There are some limitations to claims data collection in randomized phase III trials. First, expenditures in a clinical trial and daily medical practice may not be the same. This may affect the generalizability of results [27]. However, we believe that the influence was similar in both arms. Second, in this trial, claims data were received from each institution with patient approval. As such, it was difficult to collect data if patients had received treatment from other clinics or hospitals, or changed their hospitals. For example, some patients might have been transferred to another institution to receive terminal care, but such data could not be collected. Although costs of terminal care may differ between the two groups, in many cases with cancer, most procedures are provided by experts; therefore, costs of cancer treatment provided by non-experts (i.e., other clinics and hospitals) can be regarded as 
unrelated medical costs [28]. Although there remains controversy about the handling of unrelated medical costs, the Japanese economic evaluation guideline [20] recommends that these costs should not be included in treatment costs. Lastly, claims data from pharmacies could not be collected for the same reason. Instead, we recorded prescribed drugs, which were then included in the costs of drugs. Calculating pharmacy fees in Japan is complicated (e.g., it depends on the type of pharmacy), and it is difficult to predict exact fees based only on the information provided by claims and prescription data. In this analysis, pharmacy fees were not included, although the standard pharmacy fee for 28 days of S-1 use ranges approximately from JPY 2000 to JPY 2500 based on a simple calculation. This was not reflected in our results.

The time horizon of our analysis was limited to 4 years. We believe this period is long enough to evaluate the cost-effectiveness of S-1 therapy. Normally, in an economic evaluation, a survival curve is estimated parametrically and extrapolated to obtain an estimated curve; the expected survival time or other measures are calculated using this curve. In the present analysis, the results were not changed even when the survival curve was extrapolated. Therefore, we used a more robust non-parametric Kaplan-Meier method without extrapolation.

The SELECT BC trial is one of the first oncology studies in Japan that collected EQ-5D and claims data continuously over a long period. The present analysis mainly used data from this trial, which enabled a robust analysis, and demonstrated that it is highly likely that oral S-1 therapy is cost-effective. In the area of outcomes research, attention is focused on real-world data (e.g., registry, claims database), although results sometimes have internal validity issues (even if external validity is high) when compared between two different treatment groups. We believe that trial- and real-world-based methods are complementary to each other, and even if studies based on real-world data increase due to improved availability of such data, the importance of trialbased analysis, such as the present study, should not be underestimated.

\section{Conclusions}

Our results show that the introduction of oral S-1 therapy for metastatic breast cancer is cost-effective with a high probability. S-1 demonstrates potential for becoming a standard therapy for first-line metastatic breast cancer treatment in comparison with taxenes from the perspective of cost-effectiveness.

\section{Appendix}

Table 5 Detailed results of EQ-5D analysis based on a mixed linear model

\begin{tabular}{|c|c|c|c|c|c|}
\hline Effect & Group & Month & Estimate & Standard error & $p$-value \\
\hline Intercept & & & 0.3030 & 0.06016 & $<.0001$ \\
\hline Baseline & & & 0.4947 & 0.04474 & $<.0001$ \\
\hline \multirow[t]{2}{*}{ Group } & Taxane & & 0.0328 & 0.07435 & 0.6591 \\
\hline & $S-1$ & & reference & & \\
\hline \multirow[t]{11}{*}{ Visit } & & 3 & 0.1211 & 0.04914 & 0.0139 \\
\hline & & 6 & 0.0964 & 0.04925 & 0.0505 \\
\hline & & 12 & 0.1044 & 0.04935 & 0.0345 \\
\hline & & 18 & 0.1117 & 0.04956 & 0.0243 \\
\hline & & 24 & 0.0803 & 0.04975 & 0.1067 \\
\hline & & 30 & 0.0491 & 0.05033 & 0.3297 \\
\hline & & 36 & 0.0615 & 0.05138 & 0.2317 \\
\hline & & 42 & 0.0428 & 0.05324 & 0.4219 \\
\hline & & 48 & -0.0014 & 0.05494 & 0.9793 \\
\hline & & 54 & -0.0036 & 0.05813 & 0.9504 \\
\hline & & 60 & reference & . & . \\
\hline \multirow[t]{11}{*}{ Group*visit } & Taxane & 3 & -0.0671 & 0.07489 & 0.3701 \\
\hline & & 6 & -0.0600 & 0.07500 & 0.4238 \\
\hline & & 12 & -0.0831 & 0.07527 & 0.2699 \\
\hline & & 18 & -0.0697 & 0.07578 & 0.3579 \\
\hline & & 24 & -0.0553 & 0.07598 & 0.4665 \\
\hline & & 30 & -0.0228 & 0.07681 & 0.7665 \\
\hline & & 36 & -0.0526 & 0.07774 & 0.4991 \\
\hline & & 42 & -0.0594 & 0.08048 & 0.4603 \\
\hline & & 48 & -0.0005 & 0.08314 & 0.9951 \\
\hline & & 54 & 0.0294 & 0.08627 & 0.7334 \\
\hline & & 60 & reference & . & . \\
\hline
\end{tabular}

\section{Abbreviations}

BNF: British National Formulary; Cl: Confidence interval; EORTC QLQ-C30: The European Organization for Research and Treatment of Cancer Core Quality of Life Questionnaire C30; EQ-5D: EuroQol 5-dimension; FAS: Full analysis set; HR: Hazard ratio; HRQOL: Health-related quality of life; HTA: Health technology assessment; ICER: Incremental cost-effectiveness ratio; MBC: Metastatic breast cancer; MMRM: Mixed models for repeated measures; NICE: National Institute for Health and Care Excellence; OS: Overall survival; PBAC: Pharmaceutical Benefits Advisory Committee; pCODR: The panCanadian Oncology Drug Review; PFS: Progression free survival; QALY: Quality-adjusted life year; RCT: Randomized controlled trial; UMIN: University Hospital Medical Information Network; WTP: Willingness to pay

\section{Acknowledgements}

This study was sponsored by the Comprehensive Support Project for Oncology Research (CSPOR) of the Public Health Research Foundation. The research fund was provided to CSPOR by Taiho Pharmaceutical Co., Ltd. under the study contract. Taiho Pharmaceutical took no part in this study other than providing information relevant to the proper use of the study drug; i.e., they had no input into design or analysis of the cost-effectiveness analysis, nor 
were they involved in the final review. We also gratefully acknowledge the support from Comprehensive Support Project for Health Outcomes Research (CSP-HOR).

\section{Funding}

Comprehensive Support Project for Oncology Research (CSPOR) of the Public Health Research Foundation. CSPOR had no role in the design of the study, data collection and analysis, interpretation of data and writing the manuscript.

\section{Availability of data and materials}

The data that support the findings of this study are available from Comprehensive Support Project for Oncology Research (CSPOR) of the Public Health Research Foundation, but are not publicly available as restrictions apply to the availability of these data, which were used under license for the current study. Data are however available from the authors upon reasonable request and with permission of CSPOR.

\section{Authors' contributions}

Conception and design: ST, FT, SK, MM, OS, HY, SY, OY, MH. Acquisition of data: OS, HY, SY, MH. Analysis and interpretation of data: ST, FT, SK, MM, HY, KT, OY. Drafting the manuscript: ST. Approval of the final manuscript: All authors read and approved the final manuscript.

\section{Ethics approval and consent to participate}

This study was approved by the ethics committee of National Cancer Center Hospital East. We obtained written informed consent from all the participants.

\section{Consent for publication}

Not applicable.

\section{Competing interests}

Dr. Ohashi has stock ownership of Statcom, received honoraria from Sanofi, had a consultant/advisory role of Taiho, Chugai, Shionogi and Eisai. Dr. Mukai received honoraria from AstraZeneca, Novartis and Taiho, and research funding from Chugai, Daiichi Sankyo, Eisai, Nippon Kayaku, Novartis, Pfizer and Sanofi. All remaining authors have declared no conflicts of interest.

\section{Publisher's Note}

Springer Nature remains neutral with regard to jurisdictional claims in published maps and institutional affiliations.

\footnotetext{
Author details

'Department of Health and Welfare Services, National Institute of Public Health, 2-3-6 Minami, Wako, Saitama 351-0197, Japan. ²Department of Biomedical Sciences, College of Life Sciences, Ritsumeikan University, 1-1-1 Noji-higashi, Kusatsu, Shiga 525-8577, Japan. ${ }^{3}$ Kanagawa Academy of Science and Technology (KAST), 3-2-1 Sakado, Takatsu-ku, Kawasaki, Kanagawa 213-0012, Japan. ${ }^{4}$ Department of Biostatistics, School of Public Health, The University of Tokyo, 7-3-1 Hongo, Bunkyo-ku, Tokyo 113-0033, Japan. ${ }^{5}$ Biostatistics Division, Clinical Research Support Center, The University of Tokyo Hospital, 7-3-1 Hongo, Bunkyo-ku, Tokyo 113-0033, Japan. ${ }^{6}$ Department of Breast Oncology, National Hospital Organization Shikoku Cancer Center, 160 Kou, Minamiumemoto-machi, Matsuyama, Ehime 791-0280, Japan. ${ }^{7}$ Department of Breast and Endocrine Surgery, University of Tsukuba Hospital, 2-1-1 Amakubo, Tsukuba, Ibaraki 305-8576, Japan. ${ }^{8}$ Department of Breast Surgery, Ibaraki Prefectural Central Hospital, 6528 Koibuchi, Kasama, Ibaraki 309-1793, Japan. ${ }^{9}$ Breast Surgery Department, Social Medical Corporation Hakuaikai Sagara Hospital, Matsubara-cho 3-31, Kagoshima 892-0833, Japan. ${ }^{10}$ Department of Integrated Science and Engineering, Chuo University, 1-13-27 Kasuga, Bunkyo-ku, Tokyo 112-8551, Japan. ${ }^{11}$ Division of Breast and Medical Oncology, National Cancer Center Hospital East, 6-5-1 Kashiwanoha, Kashiwa, Chiba 277-8577, Japan.
}

Received: 19 June 2017 Accepted: 13 November 2017 Published online: 17 November 2017

\section{References}

1. Burki TK. NICE restructures the cancer drugs fund. Lancet Oncol. 2016; 17(1):e9.

2. Timmins N. At last, NICE to take over the cancer drugs fund. BMJ. 2016;352: i1324.

3. Squires H, Stevenson M, Simpson E, Harvey R, Stevens J. Trastuzumab Emtansine for treating HER2-positive, Unresectable, locally advanced or metastatic breast cancer after treatment with Trastuzumab and a Taxane: an evidence review group perspective of a NICE single technology appraisal. PharmacoEconomics. 2016:34(7):673-80.

4. Wade R, Duarte A, Simmonds M, Rodriguez-Lopez R, Duffy S, Woolacott N, Spackman E. The clinical and cost effectiveness of Aflibercept in combination with Irinotecan and fluorouracil-based therapy (FOLFIRI) for the treatment of metastatic colorectal cancer which has progressed following prior Oxaliplatin-based chemotherapy: a critique of the evidence. PharmacoEconomics. 2015;33(5):457-66

5. Greenhalgh J, Bagust A, Boland A, Oyee J, Trevor N, Beale S, Dundar Y, Hockenhull J, Proudlove C, O'Reilly S. Eribulin for the treatment of advanced or metastatic breast cancer: a NICE single technology appraisal. PharmacoEconomics. 2015;33(2):137-48

6. Maehara Y. S-1 in gastric cancer: a comprehensive review. Gastric Cancer. 2003;6(Suppl 1):2-8.

7. Takashima T, Mukai H, Hara F, Matsubara N, Saito T, Takano T, Park Y, Toyama T, Hozumi Y, Tsurutani J, et al. Taxanes versus S-1 as the first-line chemotherapy for metastatic breast cancer (SELECT BC): an open-label, noninferiority, randomised phase 3 trial. Lancet Oncol. 2016;17(1):90-8.

8. Hortobagyi GN. Treatment of breast cancer. N Engl J Med. 1998;339(14): 974-84.

9. Gradishar WJ, Anderson BO, Balassanian R, Blair SL, Burstein HJ, Cyr A, Elias AD, Farrar WB, Forero A, Giordano SH, et al. Breast cancer, version 1.2016. J Natl Compr Cancer Netw. 2015;13(12):1475-85.

10. Brooks R. EuroQol: the current state of play. Health Policy. 1996;37(1):53-72.

11. Tsuchiya A, Ikeda S, Ikegami N, Nishimura S, Sakai I, Fukuda T, Hamashima C, Hisashige A, Tamura M. Estimating an EQ-5D population value set: the case of Japan. Health Econ. 2002;11(4):341-53.

12. Ramsey S, Willke R, Briggs A, Brown R, Buxton M, Chawla A, Cook J, Glick H, Liljas B, Petitti D, et al. Good research practices for cost-effectiveness analysis alongside clinical trials: the ISPOR RCT-CEA task force report. Value Health. 2005;8(5):521-33.

13. Ramsey SD, Willke RJ, Glick H, Reed SD, Augustovski F, Jonsson B, Briggs A, Sullivan SD. Cost-effectiveness analysis alongside clinical trials II-an ISPOR good research practices task force report. Value Health. 2015;18(2):161-72.

14. Weinstein MC, O'Brien B, Hornberger J, Jackson J, Johannesson M, McCabe C, Luce BR. Principles of good practice for decision analytic modeling in health-care evaluation: report of the ISPOR task force on good research practices-modeling studies. Value Health. 2003;6(1):9-17.

15. Sonnenberg FA, Beck JR. Markov models in medical decision making: a practical guide. Med Decis Mak. 1993;13(4):322-38.

16. Aaronson NK, Ahmedzai S, Bergman B, Bullinger M, Cull A, Duez NJ, Filiberti A, Flechtner H, Fleishman SB, de Haes JC, et al. The European Organization for Research and Treatment of cancer QLQ-C30: a quality-of-life instrument for use in international clinical trials in oncology. J Natl Cancer Inst. 1993; 85(5):365-76.

17. Rasanen P, Roine E, Sintonen $H$, Semberg-Konttinen V, Ryynanen OP, Roine $R$. Use of quality-adjusted life years for the estimation of effectiveness of health care: a systematic literature review. Int J Technol Assess Health Care. 2006:22(2):235-41.

18. Wisloff T, Hagen G, Hamidi V, Movik E, Klemp M, Olsen JA. Estimating QALY gains in applied studies: a review of cost-utility analyses published in 2010. PharmacoEconomics. 2014;32(4):367-75.

19. Aihara T, Toyama T, Takahashi M, Yamamoto Y, Hara F, Akabane H, Fujisawa T, Ishikawa T, Nagai S, Nakamura R, et al. The Japanese breast cancer society clinical practice guideline for systemic treatment of breast cancer, 2015 edition. Breast cancer (Tokyo, Japan). 2016;23(3):329-42.

20. Shiroiwa T, Fukuda T, Ikeda S, Takura T, Moriwaki K. Development of an official guideline for the economic evaluation of drugs/medical devices in Japan. Value Health. 2017;20(3):372-8. 
21. Lin DY, Feuer EJ, Etzioni R, Wax Y. Estimating medical costs from incomplete follow-up data. Biometrics. 1997;53(2):419-34.

22. Lord J, Asante MA. Estimating uncertainty ranges for costs by the bootstrap procedure combined with probabilistic sensitivity analysis. Health Econ. 1999;8(4):323-33.

23. Briggs $A H, O$ 'Brien BJ. The death of cost-minimization analysis? Health Econ. 2001;10(2):179-84.

24. Shiroiwa T, Sung YK, Fukuda T, Lang HC, Bae SC, Tsutani K. International survey on willingness-to-pay (WTP) for one additional QALY gained: what is the threshold of cost effectiveness? Health Econ. 2010;19(4):422-37.

25. WHO Collaborating Centre for Drug Statistics Methodology: Guidelines for ATC classification and DDD assignment, 6th edition edn. Oslo; 2003.

26. Shiroiwa T, Fukuda T, Shimozuma K, Mouri M, Hagiwara Y, Doihara H, Akabane H, Kashiwaba M, Watanabe T, Ohashi Y, et al. Long-term health status as measured by EQ-5D among patients with metastatic breast cancer: comparison of first-line oral S-1 and taxane therapies in the randomized phase III SELECT BC trial. Qual Life Res. 2017;26(2):445-53.

27. Sculpher MJ, Claxton K, Drummond M, McCabe C. Whither trial-based economic evaluation for health care decision making? Health Econ. 2006; 15(7):677-87.

28. Morton A, Adler Al, Bell D, Briggs A, Brouwer W, Claxton K, Craig N, Fischer $A, M c G r e g o r ~ P$, van Baal P. Unrelated future costs and unrelated future benefits: reflections on NICE guide to the methods of technology appraisal. Health Econ. 2016;25(8):933-8.

\section{Submit your next manuscript to BioMed Central} and we will help you at every step:

- We accept pre-submission inquiries

- Our selector tool helps you to find the most relevant journal

- We provide round the clock customer support

- Convenient online submission

- Thorough peer review

- Inclusion in PubMed and all major indexing services

- Maximum visibility for your research

Submit your manuscript at www.biomedcentral.com/submit 